\title{
Existence and Uniqueness of Weak Solution for chemotaxis model coupled with heat equation
}

\author{
Ali slimani ${ }^{1}$ and Amar Guesmia ${ }^{1}$ \\ ${ }^{1} 20$ August 1955 University of Skikda
}

April 4, 2021

\begin{abstract}
Keller-Segel chemotaxis model is described by a system of nonlinear PDE : a convection diffusion equation for the cell density coupled with a reaction-diffusion equation for chemoattractant concentration. In this work, we study the phenomenon of Keller Segel model coupled with a heat equation, because The heat has an effect the density of the cells as well as the signal of chemical concentration, since the heat is a factor affecting the spread and attraction of cells as well in relation to the signal of chemical concentration, The main objectives of this work is the study of the global existence and uniqueness and boundedness of the weak solution for the problem defined in (8) for this we use the technical of Galerkin method.
\end{abstract}

\section{Hosted file}

Slimani-paper.pdf available at https://authorea.com/users/400771/articles/516642-existenceand-uniqueness-of-weak-solution-for-chemotaxis-model-coupled-with-heat-equation 